ARTIGOS / ARTICLES

DOI: $10.5433 / 1679-0367.2022 v 43 n 1 p 75$

\title{
Automedicação em usuários da Atenção Primária à Saúde: motivadores e fatores associados
}

\author{
Self-medication in users of Primary Health Care: \\ motivators and associated factors
}

\author{
Rafael Openkowski Ramires ${ }^{1}$, Ivana Loraine Lindemann ${ }^{2}$, \\ Gustavo Olszanski Acrani ${ }^{3}$, Lissandra Glusczak ${ }^{4}$
}

Resumo

\begin{abstract}
Introdução: a automedicação pode aliviar sintomas e doenças agudas por um menor custo. Entretanto, esse consumo de medicamentos por conta própria e sem orientação adequada pode acarretar prejuízos como terapêuticas inadequadas, intoxicações e dependência. Objetivo: estimar a prevalência, os motivadores e os fatores associados à automedicação em adultos e idosos atendidos na Atenção Primária à Saúde (APS). Métodos: estudo transversal realizado de maio a agosto de 2019. A estatística compreendeu o cálculo da prevalência de automedicação, com período recordatório de 30 dias e seu intervalo de confiança de 95\% (IC 95\%). Para verificação dos fatores ajustados, foram calculadas as Razões de Prevalência (RP), brutas e ajustadas. Resultados: a amostra foi de 1.365 usuários, com prevalência do desfecho de 55\% (IC 95\%: 53-58), sendo esta maior em mulheres $(\mathrm{RP}=1,33$; IC 95\%: 1,17-1,52), adultos ( $\mathrm{RP}=1,27$; IC 95\%: 1,14-1,41) e naqueles com 12 anos ou mais de estudo ( $\mathrm{RP}=1,22$; IC 95\%: 1,09-1,37). Os principais motivadores foram dor (89\%), gripe, resfriado e dor de garganta $(18,9 \%)$ e febre $(6,9 \%)$. Conclusão: verificou-se prevalência importante de automedicação, especialmente em mulheres, jovens e com maior escolaridade. Considerando os riscos, destaca-se a necessidade de políticas públicas para prevenir o uso indiscriminado de medicamentos.
\end{abstract}

Palavras-chave: Automedicação; Atenção Primária à Saúde; Epidemiologia.

\footnotetext{
${ }^{1}$ Graduando em Medicina na Universidade Federal da Fronteira Sul (UFFS), Passo Fundo, Rio Grande do Sul, Brasil.

${ }^{2}$ Doutorado em Ciências da Saúde pela Universidade Federal do Rio Grande (FURG), Rio Grande, Rio Grande do Sul, Brasil. Professora Adjunta na Universidade Federal da Fronteira Sul, Passo Fundo, Rio Grande do Sul, Brasil.

${ }^{3}$ Doutorado em Biologia Celular e Molecular pela Faculdade de Medicina de Ribeirão Preto da Universidade de São Paulo (FMRP-USP), Ribeirão Preto, São Paulo, Brasil. Professor na Universidade Federal da Fronteira Sul, Passo Fundo, Rio Grande do Sul, Brasil.

${ }^{4}$ Doutorado em Ciências Biológicas (Bioquímica Toxicológica) pela Universidade Federal de Santa Maria (UFSM), Santa Maria, Rio Grande do Sul, Brasil. Professora Associada D na Universidade Federal da Fronteira Sul, Passo Fundo, Rio Grande do Sul, Brasil.E-mail: lissandra.glusczak@uffs.edu.br
} 


\begin{abstract}
Introduction: self-medication can relieve symptoms and diseases at a lower cost. However, this can lead to losses such as inappropriate therapies, intoxications and dependence. Objective: to estimate the prevalence, motivators and factors associated with self-medication in adults and the elderly treated in Primary Health Care. Methods: cross-sectional study carried out from May to August 2019. The statistics comprised the calculation of the prevalence of self-medication, with a recall period of 30 days and its $95 \%$ confidence interval (95\% CI). In order to check the adjusted factors, crude and adjusted Prevalence Ratios (PR) were calculated. Results: the sample consisted of 1,365 users, with an outcome prevalence of 55\% (95\% CI: 53-58), which was higher in women $(\mathrm{PR}=1.33 ; 95 \% \mathrm{CI}: 1.17-1.52)$, adults $(\mathrm{PR}=1.27 ; 95 \% \mathrm{CI}: 1.14-1.41)$ and in those with 12 or more years of study ( $\mathrm{PR}=1.22 ; 95 \% \mathrm{CI}: 1.09-1.37)$. The main motivators were pain (89\%), flu, cold and sore throat (18.9\%) and fever (6.9\%). Conclusion: there was an important prevalence of self-medication, especially in women, young people and those with higher education. Considering the risks, the need for public policies to prevent the indiscriminate use of medicines is highlighted.
\end{abstract}

Keywords: Self-medication; Primary Health Care; Epidemiology.

\section{Introdução}

Medicamentos, segundo a Agência Nacional de Vigilância Sanitária (Anvisa), são produtos que visam aliviar sintomas e prevenir ou curar doenças, bem como auxiliar nos diagnósticos. ${ }^{(1)}$ Ainda, a Organização Mundial da Saúde (OMS) define automedicação como o uso de fármacos sem prescrição ou supervisão de médico ou dentista. ${ }^{(2)}$ No Brasil, são legalmente habilitados para prescrever medicamentos para seres humanos: os médicos; os cirurgiões dentistas para uso unicamente odontológico; e os farmacêuticos em relação a fármacos isentos de prescrição. ${ }^{(3)}$ Inclusive, o enfermeiro, como membro integrante da equipe de saúde, dispõe de amparo ético-legal para prescrever certos medicamentos, quando dentro dos limites que a própria Lei do Exercício Profissional de Enfermagem (Lei no 7.498, de 25 de junho de 1986) impõe, tais como as normatizações do Ministério da Saúde (MS) e as resoluções do Conselho Federal de Enfermagem (Cofen) que regulamentam essa atividade. $^{(4)}$

Destaca-se que o consumo de medicamentos sem orientação, se de forma racional pode trazer benefícios tanto para a saúde dos seus usuários, quanto para a economia do sistema de saúde, ${ }^{(5-6)}$ no entanto, o uso de fármacos com base somente em conhecimentos empíricos pode ocasionar terapêuticas inadequadas, possível dependência e também efeitos colaterais indesejáveis. ${ }^{(7)}$

Mesmo em doses padronizadas, nenhum fármaco está isento de efeitos adversos. Por exemplo, os anti-inflamatórios não esteroidais (AINEs) estão associados a distúrbios gástricos. ${ }^{\left({ }^{8}\right)}$ Além disso, devido à farmacogenômica, cada indivíduo responde de maneira diferente ao mesmo tratamento, o que pode levar à não resposta e a efeitos colaterais preocupantes. ${ }^{(9)}$ Inclusive, medicamentos são considerados os principais causadores de intoxicações no Brasil, ${ }^{(10)}$ os quais sofrem metabolismo, principalmente hepático e renal, e uma vez que não bem tolerados poderão lesionar tais sistemas. ${ }^{(11)}$

Muitos fármacos contêm componentes com potencial de agravar condições clínicas já existentes ou de ocasionar efeitos devido a interações medicamentosas. Cita-se, nesse contexto, produtos para resfriado ou tosse que possuem efeitos simpatomiméticos, devendo ser usados com cautela por hipertensos, diabéticos com insulinoterapia ou pessoas com hipertireoidismo. Também, os sprays nasais, se usados por mais de quatro dias podem gerar uma congestão rebote; remédios de uso tópico que podem causar reações alérgicas; e antiácidos 
estomacais que podem provocar constipação ou acelerar a motilidade gastrointestinal. ${ }^{(12)}$

A automedicação está relacionada à não adesão às orientações médicas e de saúde, em que a pessoa ou seu responsável determina o melhor tratamento. ${ }^{(13-15)}$ Tal prática é fomentada por fatores como a facilidade de compra e/ou utilização, bem como a quantidade massiva de propaganda de medicamentos contrastando com poucas campanhas quanto aos riscos. ${ }^{(16)}$ Assim, se por um lado os fármacos são úteis à manutenção e recuperação da saúde, por outro lado, o seu uso irracional tem gerado um cenário preocupante dia após dia. ${ }^{(17)}$

Ressalta-se que, apesar dos perigos dessa prática, há poucos estudos sobre a temática no Brasil. ${ }^{(8)}$ Dentre os trabalhos disponíveis, nacionais e internacionais, a prevalência de automedicação foi de aproximadamente $15 \%$, referindo-se a um período de sete dias, e de até quase $90 \%$ no período de um ano. Para 30 dias foi encontrada uma variação entre $38,8 \%$ e $55 \% .^{(18-21)}$ Ademais, a maior frequência de uso foi em mulheres e jovens, e os principais motivadores foram a dor e as infecções de vias aéreas superiores. ${ }^{(8,14,16,19)}$

Contudo, poucos estudos abordaram populações atendidas pela Atenção Primária à Saúde (APS) relacionando-as com a prática da automedicação nos últimos 30 dias. Assim, tal avaliação poderá contribuir com a garantia de promoção, proteção, tratamento e recuperação da saúde de forma adequada. ${ }^{(22)}$ Diante do exposto, este estudo objetivou determinar a prevalência da automedicação em usuários da APS, bem como identificar fatores associados, os quais são aqueles que podem deixar as pessoas vulneráveis à automedicação, e os principais motivadores, que são os sinais e sintomas que levaram a tal prática.

\section{Metodologia}

O estudo faz parte de uma pesquisa transversal, realizada de maio a agosto de 2019, com usuários do Sistema Único de Saúde (SUS), atendidos nas 34 unidades urbanas da Atenção Primária à Saúde do município de Passo Fundo, no estado do Rio Grande do Sul, Brasil.

Para a definição do tamanho da amostra foi considerado um nível de confiança de $95 \%$ e um poder de estudo de $80 \%$. Tendo como base uma razão de não expostos/expostos de 9:1, prevalência total do desfecho de $10 \%$, frequência esperada do desfecho em não expostos de $9,1 \%$, e Razões de Prevalência (RP) de 2, seriam necessários 1.220 entrevistados. Acrescentando-se 15\% para fatores de confusão, a amostra necessária seria de 1.403 participantes. Foram incluídos usuários com 18 anos ou mais, que residissem na cidade de Passo Fundo, e excluídos quem estivesse impossibilitado de responder o questionário, assim como as pacientes gestantes.

Para definir o número de entrevistados por local, utilizou-se, proporcionalmente, o quantitativo de procedimentos realizados em cada unidade no mês anterior ao início da coleta de dados. Os dados foram coletados por meio de questionário testado e pré-codificado, aplicado por entrevistadores treinados, respeitando o funcionamento habitual dos locais. Foram incluídos de forma consecutiva todos os usuários que estavam na unidade para a realização de algum procedimento, até que se atingisse o " $n$ " necessário em cada local, ou até que todos os presentes no último turno da coleta fossem convidados a participar.

O desfecho estudado, "prática de automedicação", foi aferido por meio da pergunta: "nos últimos 30 dias, você tomou algum remédio por conta própria, sem receita?". Em caso de resposta afirmativa, se perguntava: "para que você tomou remédio?”. As opções de resposta (não lidas aos participantes) incluíram febre; gripe, resfriado e dor de garganta; dor; problemas digestivos; cólicas menstruais; e outros problemas, sendo, neste caso, perguntado quais.

As variáveis sobre características demográficas e socioeconômicas contemplaram: sexo; idade (em anos completos, categorizada em 18-59 e $\geq 60$ ); cor da pele autorreferida (branca, outras); escolaridade (anos de estudo: $\leq 8,9-11, \geq 12$ ); situação 
conjugal (com, sem cônjuge); e renda mensal familiar per capita em salários mínimos (valor de $\mathrm{R} \$ 998,00$ à época, $>1, \leq 1)$. Quanto às características de saúde, foram avaliados autopercepção da saúde (positiva, negativa); excesso de peso (a partir de peso e altura autorreferidos e classificação pelo Índice de Massa Corporal (IMC); ${ }^{(23-25)}$ multimorbidade (considerando duas ou mais doenças crônicas por diagnóstico médico autorreferido de obesidade, hipertensão arterial sistêmica, diabetes mellitus, hipercolesterolemia, hipertrigliceridemia e doença cardíaca); uso de medicação contínua; dor crônica (com duração de seis meses ou mais); vigência de tratamento psicológico; e uso de medicamento para dormir. E em relação às características comportamentais perguntou-se sobre hábito de acessar a internet; tabagismo; consumo de bebida alcoólica; e prática de atividade física.

Após dupla digitação e validação dos dados, a estatística incluiu a descrição da amostra e o cálculo da prevalência do desfecho com intervalo de confiança de 95\% (IC 95\%). As associações entre a automedicação e as variáveis preditoras foram examinadas empregando-se modelo de Regressão de Poisson com variância robusta para conglomerados. Na análise bivariada foram geradas as Razões de Prevalência (RP) brutas e seus IC 95\% e, posteriormente, procedeu-se à análise multivariada, originando as Razões de Prevalência ajustadas e seus IC 95\%. Esta análise, do tipo backward stepwise, seguiu um modelo hierárquico ${ }^{(26)}$ prédefinido, com três níveis de determinação, a saber: $1^{\circ}$ : características demográficas e socioeconômicas, $2^{\circ}$ : características de saúde, e $3^{\circ}$ : características comportamentais; em cada nível as variáveis foram ajustadas entre si, e aquelas que apresentaram valores de $\mathrm{p} \leq 0,20$ foram mantidas para ajuste com o nível seguinte. No caso das categóricas politômicas, quando houve ordenamento entre as categorias foi realizado o teste de Wald para tendência linear, e quando não houve ou não foi significativo, foi testada a heterogeneidade. Em todos os testes foi admitido erro $\alpha$ de $5 \%$, sendo considerados significativos valores de $\mathrm{p}<0,05$, para testes bicaudais.
O protocolo deste estudo foi aprovado pelo Comitê de Ética em Pesquisa com Seres Humanos da Universidade Federal da Fronteira Sul (UFFS), em Passo Fundo, Rio Grande do Sul, obedecendo a Resolução no 466/2012 do Conselho Nacional de Saúde (CNS), sob parecer de no 3.219.633.

\section{Resultados}

Os dados da Tabela 1 evidenciam as características da amostra constituída por 1.365 usuários, demonstrando predomínio de mulheres $(69,4 \%)$; adultos $(70,4 \%)$; cor da pele branca $(64,8 \%)$; até oito anos de escolaridade (47,1\%); participantes com cônjuge $(71,3 \%)$; e com renda de até um salário mínimo (70,7\%). Observaram-se 52,1\% de autopercepção positiva da saúde; $65 \%$ de excesso de peso; $42,3 \%$ de multimorbidade; $64,5 \%$ de uso de medicação contínua; $56,1 \%$ de dor crônica; $8,7 \%$ de tratamento psicológico; e $15,1 \%$ de uso de medicamento para dormir. Ainda, o hábito de acessar a internet foi mencionado por $67,9 \%$; e $81,1 \%, 69,6 \%$ e $56,9 \%$, respectivamente, negaram tabagismo, consumo de bebida alcoólica e prática de atividade física.

A automedicação nos últimos 30 dias foi relatada por 55\% (IC 95\%: 53-58) dos usuários entrevistados, os quais apontaram como principais motivadores dor (89\%); gripe, resfriado e dor de garganta $(18,9 \%)$; febre $(6,9 \%)$; e problemas digestivos $(6,2 \%)$. Também foram citadas: cólica menstrual $(2,8 \%)$; alergia $(2,4 \%)$; e ansiedade $(1,1 \%)$, entre outros motivadores (Tabela 2).

$\mathrm{Na}$ análise hierarquizada demonstrada na Tabela 3, após ajuste, mantiveram associação estatisticamente significativa apenas três variáveis do primeiro nível: sexo, idade e escolaridade. Foi evidenciada maior prevalência de automedicação em mulheres ( $R P=1,33$; IC 95\%: 1,17-1,52) e em adultos ( $\mathrm{RP}=1,27$; IC 95\%: 1,14-1,41). Em relação à escolaridade, verificou-se tendência de aumento da probabilidade do desfecho conforme acréscimo de tempo de estudo, sendo RP=1,22; IC 95\%: 1,091,37 entre aqueles com 12 anos ou mais. 
Tabela 1 - Caracterização sociodemográfica de uma amostra de adultos e idosos atendidos na Atenção Primária à Saúde (APS). Passo Fundo, Rio Grande do Sul, 2019 (n=1.365).

\section{Sexo}

Variáveis

Masculino

Feminino

Idade em anos completos $(\mathrm{n}=1.361)$

$\geq 60$

$18-59$

Cor da pele autorreferida $(\mathrm{n}=1.359)$

Outras

Branca

Escolaridade em anos $(\mathrm{n}=1.261)$

$\leq 8$

9-11

$\geq 12$

Situação conjugal ( $\mathrm{n}=1.358)$

Sem cônjuge

Com cônjuge

Renda mensal familiar per capita em SM* $(\mathrm{n}=1.278)$

$>1$

$\leq 1$

Autopercepção da saúde ( $\mathrm{n}=1.354)$

Positiva

Negativa

Excesso de peso $(\mathrm{n}=1.196)$

Não

Sim

Multimorbidade** $(\mathrm{n}=1.365)$

Não

Sim

Uso de medicação contínua ( $\mathrm{n}=1.365)$

Não

484

Sim

881

341

436

418

947

403

958

478

881

594

415

252

390

968

374

904

706

648

419

777

788

577

Dor maior crônica $(\mathrm{n}=777)$

Não

Sim
57,7

42,3

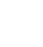

$\%$

30,6

69,4

29,6

70,4

35,2

64,8

47,1

32,9

20,0

28,7

71,3

29,3

70,7

52,1

47,9

35,0

65,0

35,5

64,5

43,9

56,1 


\section{Continuação}

Em tratamento psicológico $(\mathrm{n}=1.363)$

Não

Sim

Uso de medicamento para dormir $(\mathrm{n}=1.362)$

Não

Sim

Hábito de acessar a internet $(\mathrm{n}=1.363)$

Não

Sim

926

67,9

Tabagismo $(\mathrm{n}=1.363)$

Não

Sim

Consumo de bebida alcoólica $(\mathrm{n}=1.364)$

Não

950

69,6

Sim

414

30,4

Prática de atividade física $(\mathrm{n}=1.364)$

Sim

Não

776

56,9

Legenda: *SM: salário mínimo (R \$ 998,00). **Diagnóstico médico autorreferido de duas ou mais doenças crônicas. Fonte: os autores.

Tabela 2 - Motivadores para automedicação nos últimos 30 dias, relatados por adultos e idosos atendidos na Atenção Primária à Saúde (APS). Passo Fundo, Rio Grande do Sul, 2019 (n=756).

\begin{tabular}{lcc}
\hline Motivadores & $\mathbf{n}$ & $\mathbf{\%}$ \\
\hline Dor $(\mathrm{n}=753)$ & 670 & 89,0 \\
Gripe, resfriado e dor de garganta $(\mathrm{n}=748)$ & 141 & 18,9 \\
Febre $(\mathrm{n}=750)$ & 52 & 6,9 \\
Problema digestivo $(\mathrm{n}=750)$ & 45 & 6,2 \\
Cólica menstrual $(\mathrm{n}=750)$ & 21 & 2,8 \\
Alergia (n=756) & 18 & 2,4 \\
Ansiedade (n=756) & 8 & 1,1 \\
Inflamação (n=756) & 6 & 0,8 \\
Tensão muscular $(\mathrm{n}=756)$ & 4 & 0,5 \\
Sinusite (n=756) & 2 & 0,3 \\
Problema para dormir $(\mathrm{n}=756)$ & 2 & 0,3 \\
Infecção (n=756) & 1 & 0,1 \\
Problema cardíaco $(\mathrm{n}=756)$ & 1 & 0,1 \\
\hline
\end{tabular}

Fonte: os autores. 
Tabela 3 - Análise bruta e ajustada de fatores associados à automedicação em usuários da Atenção Primária à Saúde (APS). Passo Fundo, Rio Grande do Sul, 2019 (n=1.365).

\begin{tabular}{lllll}
\hline Variáveis & RP bruta (IC 95\%) & p & RP ajustada (IC 95\%) & p \\
\hline
\end{tabular}

$1^{0}$ nível: características demográficas e socioeconômicas $(n=1.170)$

Sexo

Masculino

Feminino

Idade em anos completos

$\geq 60$

18-59

Cor da pele autorreferida

Outras

Branca

Escolaridade em anos

$\leq 8$

9-11

$\geq 12$

Situação conjugal

Sem cônjuge

Com cônjuge

Renda mensal familiar per capita em SM*

$>1$

$\leq 1$

Autopercepção da saúde

Positiva

Negativa

Excesso de peso

Não

Sim

Multimorbidade**

Não

Sim

Uso de medicação contínua

Não

Sim

Dor crônica

Não

Sim $<0,001^{\text {a }}$

1,00

$1,38(1,23-1,55)$

1,00

$1,37(1,24-1,52)$

1,00

$1,08(0,99-1,19)$

1,00

$1,10(1,00-1,22)$

$1,31(1,15-1,48)$

1,00

$1,03(0,91-1,15)$

$0,338^{\mathrm{a}}$

1,00

$1,06(0,94-1,20)$

$2^{\circ}$ nível: características de saúde $(n=622)$

$<0,001^{\text {b }}$

$1,10(0,99-1,23)$

1,00

1,00

$1,27(1,14-1,41)$

$0,095^{\mathrm{a}}$

1,00

$1,33(1,17-1,52)$

$<0,001^{\text {a }}$

$<0,001^{\text {a }}$

$<0,001^{\text {a }}$

1,00

$1,04(0,94-1,14)$

$1,22(1,09-1,37)$

$0,656^{\mathrm{a}}$

1,00

$1,01(0,89-1,14)$

$0,414^{\mathrm{a}}$

1,00

$1,06(0,92-1,23)$

$0,089^{\mathrm{a}}$

$0,001^{\mathrm{b}}$

$0,071^{\mathrm{a}}$

$0,555^{\mathrm{a}}$

1,00

$1,09(0,99-1,20)$

1,00

$1,03(0,94-1,13)$

$0,371^{\text {a }}$

1,00

$0,95(0,86-1,06)$

1,00

$0,92(0,82-1,03)$

$0,024^{\mathrm{a}}$

1,00

$0,90(0,83-0,99)$

1,00

$1,03(0,89-1,18)$

$0,001^{\text {a }}$

$0,058^{\mathrm{a}}$

1,00

$0,85(0,78-0,93)$

1,00

$0,89(0,78-1,00)$

$0,108^{\mathrm{a}}$

$0,056^{\mathrm{a}}$

1,00

$1,06(0,99-1,14)$

$1,00(1,00-1,21)$

Continua 


\begin{tabular}{|c|c|c|c|c|}
\hline Em tratamento psicológico & & $0,764^{\mathrm{a}}$ & & $0,506^{\mathrm{a}}$ \\
\hline Não & 1,00 & & 1,00 & \\
\hline Sim & $0,97(0,78-1,20)$ & & $0,92(0,73-1,17)$ & \\
\hline Uso de medicamento para dormir & & $0,575^{\mathrm{a}}$ & & $0,396^{\mathrm{a}}$ \\
\hline Não & 1,00 & & 1,00 & \\
\hline Sim & $1,05(0,89-1,22)$ & & $1,08(0,90-1,29)$ & \\
\hline \multicolumn{5}{|c|}{$3^{0}$ nível: características comportamentais $(n=626)$} \\
\hline Hábito de acessar a internet & & $<0,001^{\mathrm{a}}$ & & $0,352^{\mathrm{a}}$ \\
\hline Não & 1,00 & & 1,00 & \\
\hline Sim & $1,35(1,21-1,51)$ & & $1,07(0,93-1,22)$ & \\
\hline Tabagismo & & $0,081^{\mathrm{a}}$ & & $0,422^{\mathrm{a}}$ \\
\hline Não & 1,00 & & 1,00 & \\
\hline Sim & $1,08(0,99-1,17)$ & & $1,04(0,94-1,16)$ & \\
\hline Consumo de bebida alcoólica & & $0,251^{\mathrm{a}}$ & & $0,125^{\mathrm{a}}$ \\
\hline Não & 1,00 & & 1,00 & \\
\hline Sim & $1,06(0,96-1,17)$ & & $1,09(0,97-1,23)$ & \\
\hline Prática de atividade física & & $0,051^{\mathrm{a}}$ & & $0,585^{\mathrm{a}}$ \\
\hline Sim & 1,00 & & 1,00 & \\
\hline Não & $1,11(1,00-1,23)$ & & $0,97(0,85-1,09)$ & \\
\hline
\end{tabular}

Legenda: testes: a) qui-quadrado; b) tendência linear. *SM: salário mínimo (R $\$ 998,00)$.**Diagnóstico médico autorreferido de duas ou mais doenças crônicas. RP: Razão de Prevalência. IC 95\%: intervalo de confiança de 95\%. Fonte: os autores.

\section{Discussão}

No estudo ora apresentado, observou-se que o perfil sociodemográfico da amostra está em conformidade com outras pesquisas sobre automedicação também realizadas na APS, sendo majoritariamente compostas pelo sexo feminino e por uma população com baixas escolaridade e renda. ${ }^{(7,27)}$ Acrescenta-se que outros autores, ao avaliarem o perfil dos usuários do SUS, também demonstraram o predomínio de mulheres. ${ }^{(28)}$

Quando questionados sobre automedicação nos últimos 30 dias antes da entrevista, $55 \%$ dos participantes responderam afirmativamente. Similarmente, em outras avaliações, com o mesmo tempo retrospectivo e também realizadas com usuários do SUS, verificou-se 53\% em idosos do município de Sorocaba, estado de São Paulo, Brasil, ${ }^{(18)}$ e $38,8 \%$ em adultos e idosos do País. ${ }^{(19)}$
$\mathrm{Na}$ literatura, há uma grande discrepância de gênero, idade, escolaridade, tamanho da amostra e período do ano do estudo entre as populações estudadas, bem como em relação ao tempo prévio de automedicação analisado. Pesquisas que avaliaram período menor, por exemplo, sete dias antes da entrevista, verificaram automedicação em 14,9\% de pessoas entre 18 e 65 anos, residentes no Distrito Federal, Brasil, ${ }^{(29)}$ ao passo que em um trabalho realizado com habitantes de diversos municípios brasileiros e de várias faixas etárias, 16,1\% admitiram a prática nos últimos 15 dias. ${ }^{(8)}$ Ademais, uma revisão sistemática produzida com 21 pesquisas publicadas na Etiópia, apontou $36,8 \%$ de automedicação, variando de $12,8 \%$ a $77,1 \%$, com períodos recordatórios de duas semanas a seis meses em relação às datas das entrevistas. ${ }^{(30)}$

Já em relação a períodos maiores de tempo, como um ano, o predomínio de automedicação foi 
de $88 \%$ em idosos atendidos em uma unidade de Estratégia de Saúde da Família (ESF) no município de Diamantina, estado de Minas Gerais, Brasil. ${ }^{(7)}$ Estudo semelhante, em Unidade Básica de Saúde (UBS), com adultos entre 20 e 59 anos residentes no município de Campos Novos, estado de Santa Catarina, Brasil, verificou prevalência de $71 \%$. $^{(27)}$ No município de Natal, estado do Rio Grande do Norte, Brasil, uma pesquisa realizada com idosos que utilizam os serviços da APS demonstrou a prevalência de $66,7 \%$. ${ }^{(31)}$ Ademais, tal prática varia entre os idosos residentes em diversas localidades, como, por exemplo, nos Estados Unidos, onde se verificou que $42 \%$ usavam, no mínimo, um fármaco sem receita. ${ }^{(21)}$ E, segundo Goh et al. (2009), no sul da Austrália a prevalência de automedicação foi de $17 \%$ em 2000-2001 e de 35,5\% em 2003-2004. ${ }^{(20)}$

Ressalta-se que em pesquisa com população brasileira acima de 16 anos, sem um período delimitado para a prática, os resultados foram: 76,2\% em 2014; 72\% em 2016; e 79\% em 2018. ${ }^{(32)}$ Estudo semelhante a esse, mas com indivíduos de 18 a 41 anos do município de Crato, estado do Ceará, Brasil, mostrou que $67,6 \%$ se automedicavam. ${ }^{(16)}$ Além do mais, outros autores relataram que em idosos atendidos em consultórios particulares de Kermanshah, no Irã, a frequência desse hábito foi de $83 \%$. ${ }^{(33)}$ Em face do exposto, comprova-se prevalência significativa de automedicação em um grande número de pessoas, independente da faixa etária e do período de tempo avaliado, pois esta faz parte dos hábitos de vida desses indivíduos.

Dentre os principais motivadores para a automedicação foram mencionados dor (89\%); gripe, resfriado e dor de garganta (18,9\%); febre (6,9\%); e problemas digestivos $(6,2 \%)$. Os resultados estão em consonância com a literatura que também aponta dor (cefaleia, odinofagia, cólicas e dores osteomusculares); febre, gripe e resfriados; e problemas digestivos..$^{(7-8,16,27,30)}$ Destaca-se que outros autores identificaram a dismenorreia como motivadora para esta prática. ${ }^{(17,34)}$ Assim, evidencia-se que a automedicação está relacionada ao tratamento de doenças e sintomas agudos que atingem a população em geral. ${ }^{(8)}$
Quanto aos fatores associados, o resultado foi semelhante ao verificado em outras investigações, sendo mais frequente em mulheres, adultos e com maior escolaridade. A associação com o sexo feminino pode estar vinculada ao fato de as mulheres serem mais acometidas por dores crônicas, como cefaleias ou, ainda, por tomarem analgésicos por questões ginecológicas. ${ }^{(8)}$ Além disso, buscam com maior frequência os serviços de saúde, tanto para aliviar sintomas agudos, quanto para manter o seu bem-estar geral, praticando o autocuidado. ${ }^{(28)}$

A prevalência aumentada em adultos pode ser justificada por serem mais ativos e por isso procurarem aliviar sintomas agudos com o intuito do rápido retorno às atividades rotineiras. Ao mesmo tempo, é provável que os idosos, mais acometidos por doenças crônicas, realizem tratamentos com acompanhamento médico, evitando, assim, o ato de se automedicar. ${ }^{(14,19)}$

Os resultados demonstraram também que quanto maior a escolaridade, maior a probabilidade da automedicação, o que se assemelha a outros trabalhos, tanto nacionais ${ }^{(8)}$ quanto de outros países. ${ }^{(33)}$ Tal observação sugere que indivíduos com essas características buscam melhor qualidade de vida e acreditam que esta se dá por meio de medicamentos. No entanto, em estudo com habitantes do Distrito Federal não foi verificada essa associação, e de acordo com os autores, quanto maior o grau de instrução, menor o índice da automedicação, em consequência do conhecimento sobre os perigos de tal prática ${ }^{(29)}$ Assim, o apresentado remete à necessidade de estudos adicionais visando melhor elucidar a relação entre as duas variáveis.

A respeito de doenças crônicas, não houve associação significativa de multimorbidade com a ingestão de fármacos por conta própria $(\mathrm{RP}=1,03$; IC 95\%: 0,89-1,18). Porém, no estudo conduzido no Distrito Federal foi constatado efeito protetor $\left(\mathrm{RP}=0,38\right.$; IC 95\%: 0,28-0,51), ${ }^{(29)}$ enquanto que entre brasileiros residentes em diferentes municípios verificou-se que as doenças crônicas agiram como um fator de risco para automedicação $(\mathrm{RP}=1,33$; IC 95\%: 1,18-1,50). ${ }^{(8)}$ Esta discordância pode ser em virtude dos diferenciados acessos a tratamentos 
médicos por cada uma dessas populações. Todavia, a automedicação deve ser evitada por pessoas com doenças crônicas, pois muitas vezes são idosos e polimedicados (uso concomitante de cinco ou mais fármacos), estando dessa maneira expostos a riscos de interações medicamentosas, bem como a erros de administração. ${ }^{(7,14,35)}$

Referente à renda também não se observou associação significativa com o desfecho, o que se assemelha aos resultados relatados por outros pesquisadores. ${ }^{\left({ }^{8,29)}\right.}$ Contudo, revisão sistemática sobre automedicação no Brasil mostrou a existência de efeito protetor nas classes mais baixas, por causa do menor poder aquisitivo, e nas classes mais altas devido ao maior conhecimento sobre os possíveis malefícios, sendo, portanto, nas classes sociais intermediárias o maior predomínio dessa prática. ${ }^{(14)}$ Em vista disso, supõe-se que indivíduos com renda inferior convivam mais com os sintomas antes de recorrer aos medicamentos, influenciados por questões culturais ou de dificuldades de acesso aos serviços de saúde, ao passo que aqueles com maior renda recorram mais facilmente aos serviços médicos antes de se automedicar.

\section{Conclusão}

Como pontos fortes deste estudo destacamse o poder estatístico adequado para as análises realizadas e a contribuição com o conhecimento sobre a automedicação, porque este é o primeiro estudo a mensurar essa prática entre usuários da APS no município, demonstrando prevalência expressiva do desfecho, apesar dos riscos inerentes. Diante disso, espera-se que os resultados possam contribuir para a adoção de políticas públicas voltadas à diminuição da automedicação, sobretudo entre mulheres, jovens, com maior escolaridade e usuários do sistema público de saúde.

São exemplos de iniciativas que podem ser adotadas para diminuir o uso de fármacos por conta própria, em especial o fornecimento de acesso maior e mais facilitado a serviços de saúde; dessa forma mantém-se os benefícios do uso de medicamentos, contudo sob menor risco. Também, são necessárias restrições quanto a propagandas de medicamentos para evitar a divulgação de informações não contidas em bula ou sem evidências científicas, além de expor adequadamente os riscos nas mensagens publicitárias; e a fiscalização da venda, especialmente se é efetuada por profissional habilitado, e se há revisões da posologia e do tempo de tratamento. Enfatiza-se principalmente a realização de campanhas de conscientização na APS, adentrando nas comunidades, em relação aos riscos e ao uso racional de medicamentos.

Cita-se como limitação desta pesquisa sua natureza transversal, com possibilidade de viés de causalidade reversa para algumas variáveis. São plausíveis ainda o viés de memória, pois os usuários podem ter subestimado ou superestimado algum dado, e também o viés de informação, devido à não comprovação de respostas, como, por exemplo, dos diagnósticos médicos autorreferidos. Além disso, pode ter ocorrido influência sazonal, pois no período do ano em que ocorreram as coletas houve maior incidência de gripe e resfriados. E, por fim, considerando que a amostra não é representativa da população, além de ter sido recrutada nos serviços de saúde e em um único município, com aspectos culturais e socioeconômicos próprios, é necessário cautela na extrapolação dos resultados.

\section{Referências}

1 Agência Nacional de Vigilância Sanitária (BR). $\mathrm{O}$ que devemos saber sobre medicamentos. [Internet]. Brasília, DF: ANVISA; 2010. [citado 2021 nov 22]. Disponível em: https://www. gov.br/anvisa/pt-br/centraisdeconteudo/ publicacoes/medicamentos/publicacoes-sobremedicamentos/o-que-devemos-saber-sobremedicamentos.pdf/view

2 World Health Organization. The Role of the pharmacist in self-care and self-medication: report of the 4th WHO Consultative Group on the Role of the Pharmacist. [Internet]. The Hague, Netherlands: WHO; 1998. [cited 2021 Nov 22]. Avaliable from: https://apps.who. int/iris/bitstream/handle/10665/65860/WHO_ DAP_98.13.pdf? sequence $=1 \&$ isAllowed $=y$ 
3 Agência Nacional de Vigilância Sanitária (BR). Resolução da Diretoria Colegiada-RDC n ${ }^{\circ} 67$, de 8 de outubro de 2007. Regulamento técnico que instituiu as boas práticas de manipulação em farmácias. Diário Oficial da União; 9 out 2007; Seção 1.

4 Oguisso T, Freitas GF. Enfermeiros prescrevendo medicamentos: possibilidades e perspectivas. Rev Bras Enferm. 2007 Mar-Abr;60 (2):141-4. doi: 10.1590/S0034-716720070002 00003 .

5 World Health Organization. Guidelines for the regulatory assessment of medicinal products for use in self-medication. [Internet]. Geneva: WHO; 2000. [cited 2021 Nov 22]. Available from: https://apps.who.int/iris/bitstream/handle/10665/66154/WHO_EDM_QSM_00.1_ eng.pdf?sequence $=1 \&$ is Allowed $=\mathrm{y}$

6 Rodrigues AC. Utilização de medicamentos isentos de prescrição e economias geradas para os sistemas de saúde: uma revisão. J Bras Econ Saúde. 2017;9(1):128-36.

7 Telles Filho PC, Almeida ÁG, Pinheiro ML. Automedicação em idosos: um problema de saúde pública. Rev Enf UERJ. 2013;21(2):197201.

8 Arrais PS, Fernandes ME, Pizzol TD, Ramos LR, Mengue SS, Luiza VL, Tavares NU, Farias MR, Oliveira MA, Bertoldi AD. Prevalência da automedicação no Brasil e fatores associados. Rev Saúde Públ. 2016;50(supl2):13s.

9 Licinio J. Farmacogenômica: oportunidades e desafios. Rev Bras Psiquiatr. 2001;23(3):122-3.

10 Sociedade Brasileira de Clínica Médica. Automedicação: os riscos de uma atitude irresponsável. [Internet]. [citado 2019 maio 8]. Disponível em: http://www.sbcm.org.br/v2/index. php/artigos/2225-automedicacao-os-riscos-deuma-atitude-irresponsavel

11 Helneck I, Camargo AL. Reações adversas a medicamentos. In: Fuchs FD, Wannmacher L. Farmacologia Clínica e Terapêutica. $5^{\mathrm{a}}$ ed. Rio de Janeiro: Guanabara Koogan; 2010. p. 124-41.
12 Horn JR. Interações medicamentosas importantes e seus mecanismos. In: Katzung BG, Trevor AJ. Farmacologia básica e clínica. 13 ${ }^{\mathrm{a}}$ ed. Rio de Janeiro: McGraw Hill Brasil; 2014. p. 1118-31.

13 Aquino DS, Barros JA, Silva MD. A automedicação e os acadêmicos da área de saúde. Ciên Saúde Colet. 2010;15(5):2533-8.

14 Delgado AF, Vriesmann LC. O perfil da automedicação na Sociedade Brasileira. Rev Saúde Desenvolv. 2018;12(11):57-75.

15 Alvim HGO, Lima MM. Riscos da automedicação. Rev JRG Estud Acad. 2019;2(4):20122019.

16 Gonçalves Júnior J, Moura SES, Dantas GCL, Lima AM, Brito WSB, Siebra BOB, et al. EL. Influência da publicidade na automedicação na população de um município brasileiro de médio porte. J Health Biol Sci. 2018 Apr-Jun; 6(2):152-5.

17. Glusczak L, Barduco ES, Haack MH, Agostini CC, Simonetti AB, Muller AR, et al. Processo formativo-reflexivo para o uso racional de medicamentos nos assentamentos do município de Pontão/RS: o relato de uma experiência à luz dos ensinamentos freireanos. Rev Guará. 2018;(10):178-89.

18 Duarte LR, Gianini RJ, Ferreira LR, Camargo MD, Galhardo SD. Hábitos de consumo de medicamentos entre idosos usuários do SUS e de plano de saúde. Cad Saúde Colet. 2012;20 (1):64-71.

19 Costa CMFN, Silveira MR, Acurcio FA, Guerra Junior AA, Guibu IA, Costa KS, et al. Utilização de medicamento pelos usuários da atenção primária do Sistema Único de Saúde. Rev Saúde Públ. 2017;51(supl2):18s.

20 Goh LY, Vitry AI, Semple SJ, Esterman A, Luszcz MA. Self-medication with over-thecounter drugs and complementary medications in South Australia's elderly population. BMC Complement Altern Med. 2009 Nov 11;9:42. doi: 10.1186/1472-6882-9-42. 
21 Qato DM, Alexander GC, Conti RM, Johnson M, Schumm P, Lindau ST. Use of prescription and over-the-counter medications and dietary supplements among older adults in the United States. JAMA. 2008; 300:2867-78.

22 Ministério da Saúde (BR). Portaria $n^{\circ} 1.820$, de 13 de agosto de 2009. Dispõe sobre os direitos e deveres dos usuários da saúde. Diário Oficial da União; 14 ago 2009; Seção 1.

23 Lipschitz DA. Screening for nutritional status in the elderly. Primary care. 2011;21(1):55-67.

24 Physical status: the use and interpretation of anthropometry. Report of a WHO Expert Committee. World Health Organ Tech Rep Ser. 1995;854:1-452.

25 World Health Organization. Anthro Plus for personal computers Manual: Software for assessing growth of the world's children and adolescents. [Internet]. Geneva: WHO; 2009. [cited 25 Jun 2020]. Disponível em: https:// cdn.who.int/media/docs/default-source/ child-growth/growth-reference-5-19-years/ anthroplus-manual.pdf? sfvrsn $=\mathrm{ddd} 24 \mathrm{~b} 2 \_0$

26 Victora CG, Huttly SR, Fuchs SC, Olinto MT. The role of conceptual frameworks in epidemiological analysis: a hierarchical approach. Int J Epidemiol. 1997;26(1):224-7.

27 Ascari RA, Ferraz L, Buss E, Rennau LR, Brum ML. Estratégia saúde da família: automedicação entre os usuários. Rev Uningá review. 2014; $18(2) ; 42-7$.

28 Silva ZP, Ribeiro MC, Barata RB, Almeida MF. Perfil sociodemográfico e padrão de utilização dos serviços de saúde do Sistema Único de Saúde (SUS), 2003-2008. Ciên Saúde Colet. 2011;16:3807-16.

29 Domingues PH, Galvão TF, Andrade KR, Araújo PC, Silva MT, Pereira MG. Prevalência e fatores associados à automedicação em adultos no Distrito Federal: estudo transversal de base populacional. Epidemiol Serv Saúde. 2017;26:319-30.

30 Ayalew MB. Self-medication practice in Ethiopia: a systematic review. Patient prefer adherence. 2017;11:401-13.
31 Silva IDD, Bezerra INM, Pimenta IDSF, Silva G, Wanderley VB, Araújo Nunes VM, Souza DLB, Piuvezam G. Acesso e implicações da automedicação em idosos na atenção primária à saúde. J Health NPEPS. 2019 Jul-Dec;4(2): 132-50.

32 Instituto de Ciência, Tecnologia e Qualidade. Pesquisa - automedicação no Brasil (2018). [Internet]. 2019 fev 12. [citado 2021 abr 02]. Disponível em: https:www.ictq.com.br

33 Jafari F, Khatony A, Rahmani E. Prevalence of self-medication among the elderly in KermanshahIran. Global J Health Sci. 2015;7(2):360-5.

34 Albusalih FA, Naqvi AA, Ahmad R, Ahmad N. Prevalence of self-medication among students of pharmacy and medicine colleges of a public sector university in Dammam City, Saudi Arabia. Pharmacy. 2017;5(3):51-64.

35 Simonetti AB, Glusczak L, Somensi ET, Acrani GO, Lindemann IL. Polifarmácia: prevalência e fatores associados em usuários da atenção primária à saúde de um município do sul do Brasil. REAS. 2021;13(5):e7453. doi: 10.25248/ REAS.e7453.2021. 